Théologiques

Théologiques

\title{
L'envers des choses : travail-fantôme des femmes et théologie du sabbat
}

\section{Pierre Goldberger}

Volume 3, numéro 2, octobre 1995

Crise du travail, crise de civilisation

URI : https://id.erudit.org/iderudit/602427ar

DOI : https://doi.org/10.7202/602427ar

Aller au sommaire du numéro

\section{Éditeur(s)}

Faculté de théologie de l'Université de Montréal

\section{ISSN}

1188-7109 (imprimé)

1492-1413 (numérique)

Découvrir la revue

\section{Citer cet article}

Goldberger, P. (1995). L'envers des choses : travail-fantôme des femmes et théologie du sabbat. Théologiques, 3(2), 105-113.

https://doi.org/10.7202/602427ar
Résumé de l'article

Cet article articule deux faces cachées de la question du travail. D’abord la condition pénible du travail actuel, pris dans l'étau d'un capitalisme néo-libéral en voie de mondialisation, et illustrée par l'« invisibilisation » du travail des femmes dans le monde malgré son rôle prépondérant. Ensuite, la relativisation du travail dans la tradition réformée ancienne, et qui trouve un écho salutaire aujourd'hui dans une théologie du sabbat. 
Theologiques 3/2 (1995) 105-113.

\title{
L'envers des choses : travail-fantôme des femmes et théologie du sabbat
}

\author{
Pierre GOLDBERGER \\ Directeur du Séminaire uni \\ Montréal
}

\section{RÉSUMÉ}

Cet article articule deux faces cachées de la question du travail. D'abord la condition pénible du travail actuel, pris dans l'étau d'un capitalisme néo-libéral en voie de mondialisation, et illustrée par l'a invisibilisation * du travail des femmes dans le monde malgré son rôle prépondérant. Ensuite, la relativisation du travail dans la tradition réformée ancienne, et qui trouve un écho salutaire aujourd'hui dans une théologie du sabbat.

On m'a invité à poser quelques jalons de réflexion théologique sur le travail, à partir d'une perspective protestante. S'il sera fait quelques allusions aux Réformateurs, c'est surtout sur la limite du travail à partir de la perspective du sabbat, c'est-à-dire du repos, que sera articulée cette brève interrogation. Le sabbat-repos comme lieu théologique fondamental, non seulement sert de référence dans la Bible contre les conditions d'aliénation et d'exploitation liées à un travail dénaturé, mais plus encore, fixe une limite au travail lui-même, dans sa positivité même. Bien plus, le sabbat révèle un Dieu autre que le Deus Faber, et son "image " n'est pas davantage appellée à être réduite à l'homo faber. Ce verrou posé au travail - à celui de Dieu comme à celui des humains - ouvre la porte au règne de la grâce, invite à la liberté qui triomphe de la nécessité. Le repos est l'accomplissement du travail et non sa simple interruption. Encore qu'il ne faille pas rêver en couleur, même théologiquemnent...

En effet, face à une vision chrétienne négative du travail traditionnellement liée à la chute, à la punition, à une souffrance méritoire, ou encore, 
dans une optique protestante puritaine, à une vertu d'obéissance à la volonté divine, il fallait retrouver le nerf biblique qui présente le travail comme co- création de l'humanité et du monde. Il faut cependant se garder d'évacuer le réel et de réfléchir théologiquement de façon trop abstraite sur le travail.

\section{Le travail au féminin}

En cette fin de $\mathrm{XX}^{\mathrm{e}}$ siècle, le capitalisme néo-libéral triomphant, lié à la nouvelle division mondiale du travail et aux mutations technologiques, se présente comme un désastre pour les masses de population qui tentent de subvenir par leur travail à leurs besoins les plus élémentaires : nourriture, logement, santé, éducation. À l'échelle mondiale, on constate l'éviction croissante d'une multitude de travailleur-es du secteur formel de l'économie, au profit d'une minorité choyée dans les secteurs de pointe et leur rejet vers les secteurs informels ou pire, vers les économies de subsistances. Ces dernières, qui sont le lot d'environ $70 \%$ de l'humanité, craquent sous le poids alors que se rérrécit ou que leur échappe l'accès à leurs ressources traditionnelles (terre, eau, forêt, habitat, etc.) et que se disloquent leurs modes d'organisation. C'est dire qu'une majorité croissante du monde connaît présentement la précarité, l'appauvrissement, la misère et donc une aggravation de ses conditions de vie et de travail, que ce soit dans les domaines de l'économie formelle ou informelle, de l'économie de marché ou encore de subsistance.

Or, plus spécifiquement, parler de l'aggravation des conditions de travail à l'échelle mondiale, et notamment dans les pays du Tiers-Monde, c'est parler de la surexploitation des femmes qui assurent plus de $60 \%$ de la production mondiale. Aborder la question du travail et de la production impose d'y entrer concrètement sous l'angle incontournable du travail au féminin. Toute autre perspective escamote la réalité. Or, vu sous cet angle, le travail se présente comme injustice.

"La moitié de la population mondiale et un tiers de la force de travail est composée de femmes qui réalisent $60 \%$ des heures de travail, mais ne gagnent que $10 \%$ du revenu salarial mondial, tout en ne possèdant que $1 \%$ de la richesse (propriété, capital, moyens de production)", souligne un collectif de femmes grecques". Elles ajoutent: "Ce que nous savions déjà, commence à être publiquement formulé : le travail des femmes leur

1 Non-Aligned Women's Movement, Women Count, Count Women's Work. Document préparatoire au Forum des femmes à Beijing 1995, Athènes, sept. 1995, p. 1. 
est volé des calculs de production ${ }^{2}$. "Suit une liste qui inclut : le soin des enfants, les soins offerts aux personnes du troisième âge et aux handicapés-es, l'économie domestique, les entreprises familiales sans salaire pour les femmes, l'inégalité des salaires entre les hommes et les femmes pour un même travail, la production agricole et l'économie de subsistance, les travaux domestiques.

En 1995, un rapport des Nations Unies sur le développement humain qui fera date, et préparé sous la direction du Dr. Mahbub ul Haq, estime que la production mondiale de 23 trillions $\$$ US ne tient pas compte de 16 trillions \$ US d'activité de travail. Sur ces 16 trillions \$US, 11 trillions \$US proviennent du travail "invisible", non rémunéré, des femmes $^{3}$. Ce phénomène est cependant loin d'être réservé au TiersMonde, puisque le Rapport conclut que les chances économiques et politiques sont universellement niées aux femmes, tant dans les pays industrialisés que dans les pays en voie de développement. Dans un article portant sur le fossé grandissant concernant la reconnaissance et la rémunération du travail des hommes et des femmes, Jodi Jacobson souligne que plus de 3 milliards de personnes vivent d'une économie de subsistance dont les deux tiers sont portés par les femmes. Citant diverses études menées en Asie, en Afrique et en Amérique latine, l'auteure conclut que la discrimination selon le sexe ("gender bias») est la cause première de la pauvreté, car sous des formes diverses, celle-ci nie à des centaines de millions de femmes l'accès à l'éducation, à la formation, à la santé, aux soins et services aux enfants, et à un statut légal. En leur refusant ce qui pourrait leur permettre de sortir de la pauvreté, on empêche les femmes de transformer leur économie de subsistance menacée en une économie où elles ne seraient plus forcées de «cannibaliser " leur ressources déclinantes ${ }^{4}$.

Jacobson relève trois présupposés qui font partie des théories et des pratiques économiques en vigueur et qui sont démentis par les faits et de nombreuses études récentes $5^{5}$ :

1. Les hommes et les femmes bénéficieraient également de la croissance économique. Or une étude du Programme de développement des

2 Ibid., p. 1.

3 UNDP, Human Development Report. New York, 1995.

4 Jodi L JACOBSON, Gender Bias - Roadblock to Sustainable Development. Paper 110, New York, Worldwatch, 1994.

5 Ibid., p. 63. 
Nations Unies établit que dans tous les pays pour lesquels il existe des données, les femmes tirent de l'arrière.

2. L'accroissement du revenu des hommes améliorerait le bien-être et le niveau de vie de toute la famille. Mais alors que dans les pays du Tiers-Monde, les femmes, par exemple, ont tendance à appliquer la totalité de leur revenu au bien-être de la famille (nourriture, santé, etc.), les hommes tendent à ne contribuer que les trois quart de celuici. De plus, les femmes produisent la majeure partie de la nourriture et assurent, en fait, la subsistance de la majorité des familles du Tiers-Monde, et ce, même lorsque les hommes ont accès à un travail rémunéré.

3. Dans les familles pauvres, les ressources seraient réparties sans égard au sexe. Des études dans plusieurs pays démontrent au contraire que les hommes et les garçons sont mieux nourris et ont davantage accès aux services de santé et d'éducation que les femmes et les filles, alors que la moyenne de travail de ces dernières varie entre 12 et 18 heures par jour, comparativement à 8 à 12 heures pour les hommes.

D'autres facteurs, indicateurs et études viennent encore alourdir ces trois présupposés erronés. La nature "invisible» du travail des femmes contribue, à l'échelle mondiale, à conforter les perceptions qui en font des personnes dépendantes plutôt que productives, des fardeaux dont on pourrait se débarrasser plutôt que les pourvoyeuses économiques principales des familles et de l'économie mondiale. Cette invisibilité du travail au féminin sous-tend tous les programmes économiques, y compris la majorité des programmes de développement, et constitue la conspiration du silence par excellence qui résulte du mariage délétère entre la structure patriarcale dominante de l'organisation du monde et le capitalisme libéral qui en régit l'économie et l'accès aux ressources nécessaires à la vie.

La réalité du travail se trouve vécue dans des contextes spécifiques liés à la classe, à la culture, à la race et au genre. Pour la majeure partie de l'humanité, le travail - formel et informel - épuise et écrase. Parmi cette humanité spoliée, les femmes, principales productrices et travailleuses, se trouvent dans une large majorité réduites à une invisibilité qui constitue un profit net et non comptabilisé pour l'économie de marché capitaliste. Sans doute l'exploitation, et donc le péché, se trouve-t-elle nichée dans ce tour de passe-passe structurel qui nie jusqu'à l'existence même de leur contribution. La réappropriation d'une vision positive de la valeur du travail passe non seulement par une transformation de ses conditions et des rapports de production, mais surtout et d'abord, par une 
reconnaissance formelle et comptable des femmes comme sujets et auteures principales des activités productives et de sustentation de la vie en societé.

Ce constat sur la persistance de l'exploitation liée au travail, donne une sonorité nouvelle aux voix qui, dans la tradition Réformée ont, d'un côté certes, développé une éthique bourgeoise du travail, mais de l'autre, et plus fondamentalement, ont préféré émettre un doute radical sur la nature salvifique du travail en cherchant du côté de la liberté, de la gratuité, c'est à dire de la grâce, les conditions subjectives d'un nouveau départ pour l'humanité 6 .

\section{La Réforme : soupçon vis-à-vis du travail et théologie du sabbat}

De façon surprenante, les premières confessions de foi réformées du XVI ${ }^{\mathrm{e}}$ siècle, Confessions de La Rochelle, helvétique, de Heidleberg et de Westminster, retentissent de cette affirmation joyeuse et confiante : « la finalité du genre humain est de rendre gloire à Dieu et de se réjouir à jamais avec Dieu ". On aurait pu s'attendre à une sombre affirmation sur les vertus du travail et de l'obéissance. Sur certains points, les Réformateurs n'innovent pas et reflètent bien leur époque. Pour Luther, le travail est une vocation de Dieu, et chaque tâche (beruf) constitue une vocation (ruf) particulière. Ceci conforte et reflète la division féodale et classiste du monde. Pour Calvin, jumeau inversé des jansénistes, le travail est un remède contre les maux engendrés par l'oisiveté, un devoir d'obéissance à la volonté de Dieu, un cadre qui discipline les passions, mais aussi une vertu nécessaire pour contribuer à la construction de la société. En tant que tel, il n'est ni moyen de pénitence et encore moins moyen de salut. Et c'est sans doute ici que la Réforme apporte une contribution distincte. Luther adopte un point de vue contraire à celui de la sagesse aristotélicienne selon laquelle l'humain est ce qu'il fait et se produit lui-même. Si cela était, nous serions, argue Luther, notre propre dieu, producteurs, créateurs et enfermés dans notre inhumanité. La " justification par la foi et non par les œuvres " délivre de l'obsession écrasante, dévorante, de tout accaparer pour se construire soi-même par ses œuvres (pouvoir, identité et salut). La grâce signifie que l'humain a été libéré - par Dieu - du pouvoir de coercition des œuvres (necessitatis operum) pour être introduit dans le domaine de la liberté pour Dieu et pour les autres. Les cuvres sont alors

6 Voir le traitement de cetre question par Jürgen MOLTMANN, "The Human Play of Liberated Mankind ", dans Theology of Play. New York, Harper \& Row, 1972, p. 45ss. 
placées sous le signe de la gratuité, et leur but est non d'atteindre la piété ou la sainteté, mais de librement célébrer Dieu, enseigne Luther dans son Traité sur la liberté chrétienne. Cette gratuité ouvre à des relations libres en société où l'humain n'est plus défini - puisque justifié par Dieu - par la puissance de son travail, par son pouvoir économique et celui de sa consommation. En fait, ces pouvoirs terrassent l'humain et l'écrasent sous la loi implacable de la nécessité où l'on doit payer sa vie de sa vie et de celle des autres. Luther, tout comme Marx, place le travail sous le règne de la nécessité, et voit que l'horizon de la liberté se situe au-delà de celuici. Pourtant ce qu'ils cherchent tous deux à réconcilier sans y parvenir, c'est à introduire le règne de la liberté à l'intérieur du travail même et non simplement dans son dépassement.

La pensée de la Réforme a tenté de tenir ensemble une double contradiction. Le règne de la liberté ne peut advenir comme le simple produit de l'œuvre humaine qui reproduit constamment sa propre aliénation. Une société, relativement libre, encourage et rend possible l'espace de la liberté, mais ne produit pas des personnes libres et conviviales, ce qui a été l'espoir déçu de nombreux marxistes. Il faut que l'humain, en faisant l'expérience d'une accepration radicale, celle de la grâce, soir libéré de la peur de devoir se sauver et se construire par lui-même, pour pouvoir s'ouvrir à des œuvres de solidarité et d'amour, ainsi que le souligne aussi Dorothée Sölle ${ }^{7}$. En plaçant les œuvres non plus sous le signe de la justification de son existence mais sous celui de la liberté de construire la communauté (la sanctification), Luther reconnait que prétendre changer les personnes sans changer les conditions de leur existence constitue une illusion.

La Réforme nous présente un paradoxe historique coûteux. D'une part, la doctrine de la justification par la grâce et la foi et non par les œuvres, arrive à point nommé, au XVI $\mathrm{X}^{\mathrm{e}}$ siècle, pour libérer les mains de la bourgeoisie marchande montante. Alors que cette classe en pleine expansion se trouve précisément à poser les fondements théoriques et pratiques de l'entrepreneurship capitaliste, elle n'a plus fondamentalement à rendre compte de l'œuvre de ses mains dont ne dépend en rien l'œuvre de son salut. D'autre part, et dans sa visée profonde, au moment même où surgit la nouvelle classe triomphante de la bourgeoisie, la Réforme lui enlève

7 Dorothée SOLLE et Shirley A. CLOYES, To Work and To Love. A Theology of Creation. Minneapolis, Fortress Press, 1984, p. 55ss. La thèse principale de l'ouvrage est que dans le travail et dans l'amour nous devenons co-createurs-trices d'une nouvelle création qui est enfin nôtre. 
la prétention à la propre justice qui pourrait résulter de ses œuvres et de ses succès. Elle fait peser un soupçon radical à l'effet que les œuvres et entreprises profitables et spectaculaires de cette bourgeoisie imbue de progrès et d'humanisme, non seulement ne soient encore et toujours trop courtes pour bâtir un salut - religieux et social - mais, bien plus, qu'elles soient en train de forger de nouvelles chaînes d'exploitation au sein même de ces succès. La justification par la grâce ne fournira donc pas le marchepied du salut à une bourgeoisie satisfaite de ses œuvres. Ce message, souvent édulcoré, n'a cessé d'être porté par l'aile radicale de la Réforme, depuis la révolte des paysans du XVIe siècle de Thomas Müntzer, jusqu'à l'Évangile social de Rauschenbuch au début de ce siècle. Le travail comme œuvre aliénante et exploitante est toujours tombé sous le soupçon radical d'une aile conséquente de la Réforme.

Plus près de nous, Jürgen Moltmann, dans un ouvrage de théologie fondamentale sur la création, trace les limites même du travail en tant que tel, qu'il soit d'ailleurs œuvre divine ou œuvre humaine ${ }^{8}$. Certes, soulignet-il, l'œuvre de création de Dieu est bonne, mais celle-ci ne s'achève pas le sixième mais bien le septième jour par le repos, le sabbat. Création et sabbat forment un tout et, fait valoir Moltmann, le travail de création de Dieu a pour finalité le repos et la jouissance de l'œuvre. Le sabbat, plus qu'une dimension ajoutée faisant suite aux six jours de travail, constitue la récapitulation er la consommation de toute l'œuvre de création. Dieu accomplit son œuvre dans, par et pour le repos.

Ce faisant, trois choses se passent : Dieu se repose de son travail, et se déploie dans sa pleine liberté tout comme son œuvre prend son identité autonome. Ensuite, Dieu se repose face à son travail et fait l'expérience critique de son œuvre. Enfin, Dieu se repose dans son travail dont il jouit. Dieu fait l'expérience de son œuvre dans une relation libre pour laquelle l'œuvre a justement été créée, pour le repos et la célébration. Aussi, chaque sabbat non seulement marque la limite du travail, mais relance le rêve d'accomplissement et rappelle, au sein d'une histoire contingente, que la création et le genre humain sont créés pour la liberté et la plénitude et non pour l'asservissement et le travail. Ainsi la bénédiction du sabbat ne provient pas de l'activité de Dieu mais bien de son repos. Le sabbat fait partie de la structure même de la création qu'il révèle, précisément lorsque cesse le travail et que l'histoire peut être alors appréhendée comme création de Dieu. Exode et sabbat vont de pair puisque c'est pour

8 Jürgen MOLTMANN, «The Sabbath: The Feast of Creation », dans God in Creation. New York, SCM Press, 1985, p. 276-296. 
la libération de l'esclavage qu'intervient Dieu pour son peuple, selon son projet de création. C'est pourquoi le sabbat restera-t-il le signe distinctif de la relation de Dieu à Israël.

Aucun exode politique, social et économique de l'oppression, de la dégradation et de l'exploitation ne mène réellement à la liberté d'un monde humain sans le sabbat, sans l'abandon de toutes les œuvres, sans la sérénité qui trouve le repos dans la présence de Dieu. Mais l'inverse est aussi vrai : les hommes et les femmes ne trouvent jamais la paix du sabbat dans la présence de Dieu, sans qu'ils/elles ne trouvent leur libération de la dépendance et de la répression, de l'inhumanité et de l'absence de Dieu. Ainsi exode et sabbat sont indivisibles 9 .

Aussi le sabbat, selon Moltmann, est révélation de Dieu, non comme Deus Faber, défini comme producteur-trice/créateur-trice, mais comme Dieu de présence en sa création. Au "commencement " même de la création, le travail, si bon soit-il, se trouve inscrit dans une finalité qui le dépasse. Dans une histoire éclatée, marquée au coin de l'exploitation et de la souffrance, le sabbat non seulement met un terme - temporaire - à celles-ci, permettant la restauration et des travailleur-es et de la nature, mais il proclame surtout que c'est pour la dignité de l'accomplissement et de la jouissance du sabbat qu'existe la création et tout ce qu'elle contient.

Le sabbat annonce la libération, le repos et la restauration pour tous et toutes - humanité, terre, vie animale, végétale et minérale - et c'est pourquoi il est limitation de l'activité, du travail, qui creuse les inégalités et sépare de Dieu. Aussi, les cycles jubilaires de l'Ancien Testament, probablement peu appliqués ( $L v 25)$, annoncent-ils l'« année de grâce " de Dieu qui redistribue à tous et toutes - personnes libres et esclaves, citoyens et étrangers, hommes et femmes, animaux et pâtures- les chances de vie. L'annulation cyclique des "dettes» et des modes de subjugation de toute nature, à tous les sept ans et à tous les 49 ans, révèle qu'il fallait s'attendre à très peu de temps, à peine deux générations, pour que l'activité humaine, personnelle et sociale, ait fait son œuvre d'exploitation et d'aliénation, et que ne se trouvent de nouveau hypothéquées la liberté, la dignité et l'autonomie des travailleurs et des moyens de production. Or, les cycles jubilaires tentaient de réinscrire la finalité de l'ordre de la création à l'intérieur même de l'histoire et de sa nécessité. Jésus, souligne Moltmann, loin d'abolir le sabbat, a radicalisé sa présence au sein de la

Ibid., p. 287. 
quotidienneté de l'histoire. La liberté du sabbat peut surgir à tout moment et en tout lieu. Qu'elle vienne, car l'on se meurt encore sous le fardeau! Au sein de l'écrasement de la production, c'est le sabbat qui allume et entretient l'espérance de la libération...

Une femme de l'Andhra Pradesh, en Inde, travaille " trois journées " par jour. Elle s'éveille à quatre heures du matin, allume le feu, traie les buffles, balaie la maison, va chercher de l'eau et nourrit toute la famille. De huit heures du matin à cinq heures de l'après midi, elle a charge de désherber des champs pour un pénible et maigre salaire. En début de soirée, elle va glâner des branches, des rameaux et des feuillages pour garder le feu allumé. Elle cherche aussi des baies et des plantes sauvages pour nourrir ses enfants et doit couper des herbages pour nourrir les buffles. Finalement, elle rentre chez elle pour cuire le repas et faire ses travaux domestiques. Cette femme travaille deux fois plus d'heures chaque jour que n'importe quel homme de son village, pour faire vivre sa famille. Cependant, elle ne possède rien de la terre sur laquelle elle s'épuise chaque jour. Chaque année, malgré tous ses efforts et tout son travail, elle se trouve, comme des millions de ses scurs, encore plus appauvrie et encore moins capable de veiller à la survie de sa famille ${ }^{10}$.

10 Jodi L. JACOBSON, op. cit., p. 61. 